\title{
Measurement-Driven Multi-Target Multi-Bernoulli Filter
}

\author{
Shijie Li (D) and Humin Lei \\ Air and Missile Defense College, Air Force Engineering University, Xian 710051, China \\ Correspondence should be addressed to Shijie Li; lishijiepaper@163.com \\ Received 18 March 2018; Revised 8 July 2018; Accepted 12 July 2018; Published 22 July 2018 \\ Academic Editor: Paolo Addesso \\ Copyright ( 2018 Shijie Li and Humin Lei. This is an open access article distributed under the Creative Commons Attribution \\ License, which permits unrestricted use, distribution, and reproduction in any medium, provided the original work is properly \\ cited. \\ A measurement-driven multi-target multi-Bernoulli (MeMBer) filter which modifies the MeMBer filter by the measurements \\ information is proposed in this paper. The proposed filter refines both the legacy estimates and the data-induced estimates of the \\ MeMBer filter. For the targets under the legacy track set, the detection probabilities derived from the measurements are employed \\ to refine the multi-target distribution. And for the targets under the data-induced track set, the multi-target distribution is further \\ improved by the modified existence probabilities of the legacy tracks. Unlike the cardinality balanced MeMBer (CBMeMBer) filter, \\ the proposed filter removes the cardinality bias in the MeMBer filter by utilizing the measurements information. Simulation results \\ show that, compared with the traditional methods, the proposed filter can improve the stability and accuracy of the estimates and \\ does not need the high detection probability hypothesis.
}

\section{Introduction}

The multi-target tracking refers to estimating the number of targets as well as their individual states in the scenario of missed detection and false alarms. The random finite set (RFS) is an effective approach to multi-target tracking, as it does not need exact data association compared with joint probabilistic data association filter and multiple hypothesis tracking filter [1]. Among the solutions based on the RFS theory, the probability hypothesis density (PHD) filter is the most well-known one [2]. The PHD filter assumes that the number of targets is Poisson distributed and approximates the multi-target Bayes recursion by propagating the first-order moment. On the basis of the PHD filter, the Cardinalized PHD (CPHD) filter propagates the first-order moment as well as the cardinality distribution without making the Poisson assumption [3]; thus the cardinality estimation of the CPHD filter is more accurate and stable than the PHD filter. Recently, further researches have been made to reduce the computational cost and improve the robustness of the PHD and CPHD filters [4-6], and the multi-sensor extensions have also been developed [7].

The MeMBer filter makes a more convincing approximation of the multi-target distribution than the PHD or CPHD filter, since it propagates the multi-target distribution as a multi-target multi-Bernoulli process. In the MeMBer filter, the tracks of targets are modeled by a multi-Bernoulli parameter set. For each target, its existence probability and probability density are estimated simultaneously; thus the MeMBer filter is a parametric approximation to the multitarget Bayes recursion. Unlike the PHD and CPHD filters, the MeMBer filter does not suffer from the problem of extraction of state estimates; however, because of the approximation made in the update step, the MeMBer filter is positively biased in cardinality. In order to fix this problem, the CBMeMBer filter was proposed [8]; the bias is removed because the existence probabilities that correspond to data-induced tracks are calculated from the exact corresponding probability generating functional (p.g.fl.). In [9], an alternative approach was proposed to remove the cardinality bias by modeling the spurious targets. The CBMeMBer filter was further improved to accommodate the unknown clutter and detection profile [10]. And to improve the estimation accuracy, the multiBernoulli smoother which consists of original CBMeMBer filter and backward smoothing was proposed in [11]. In addition, the multi-Bernoulli filter was also enhanced for multi-sensor tracking $[12,13]$, extended target tracking [14, 15], multipath multi-target tracking [16], and group object tracking [17]. 
However, both the original multi-Bernoulli filter and the improved methods above will reduce the contribution of the measurements to the posterior multi-target distribution especially when the cardinality of the legacy track is big enough. This problem was indicated in [18] and then an improved MeMBer (IMeMBer) filter which modifies the legacy estimates by the measurements was proposed; however, the derivation was not rigorous enough and it ignored the refinement of the data-induced tracks parameters.

In this paper, a measurement-driven multi-target multiBernoulli filter is proposed. In the original MeMBer and CBMeMBer filters, the updated tracks are composed of two parts, the first part represents the targets under the legacy track set, while the second part represents the targets under the data-induced track set. These two parts correspond to the individual updates of the assumed undetected targets and the joint updates of all the assumed detected targets, respectively. Therefore, when the measurements originate from a certain target with a high/low probability, the target's existence probability that corresponds to the update of the assumed undetected target should be low/high. In the MeMBer and CBMeMBer filters, however, the targets under the legacy track set are updated without the measurements set. To address this problem, the proposed filter utilizes the probabilities of measurements originating from targets to modify the updates of targets under the legacy track set. In addition, the proposed filter further refines the updates of targets under data-induced track set by the modified existence probabilities of the legacy tracks.

\section{Background}

2.1. Multi-Target Multi-Bernoulli Process. For a Bernoulli RFS $\boldsymbol{X}^{(i)}$, suppose that it is either an empty set with probability $1-r^{(i)}$ or a single element set with probability $r^{(i)}$, and the probability density function is $p^{(i)}\left(\boldsymbol{x}_{i}\right)$, then the distribution function can be given by the following [1]:

$$
\pi\left(\boldsymbol{X}^{(i)}\right)= \begin{cases}1-r^{(i)}, & \boldsymbol{X}^{(i)}=\varnothing \\ r^{(i)} p^{(i)}\left(\boldsymbol{x}_{i}\right), & \boldsymbol{X}^{(i)}=\left\{\boldsymbol{x}_{i}\right\},\end{cases}
$$

and the p.g.fl. of $\pi\left(\boldsymbol{X}^{(i)}\right)$ is

$$
G[h]=1-r^{(i)}+r^{(i)}\left\langle p^{(i)}, h\right\rangle,
$$

where $\langle p, h\rangle=\int p(\boldsymbol{x}) h(\boldsymbol{x}) d \boldsymbol{x}$.

A multi-Bernoulli RFS $\boldsymbol{X}$ is a union of independent Bernoulli RFSs, that is,

$$
\boldsymbol{X}=\bigcup_{i=1}^{M} \boldsymbol{X}^{(i)}
$$

The distribution function of $\boldsymbol{X}$ is as follows [1]:

$$
\pi\left(\left\{\boldsymbol{x}_{1}, \cdots, \boldsymbol{x}_{n}\right\}\right)=\pi(\varnothing) \sum_{1 \leq i_{1} \neq \cdots \neq i_{n} \leq M} \prod_{j=1}^{n} \frac{r^{\left(i_{j}\right)} p^{\left(i_{j}\right)}\left(\boldsymbol{x}_{j}\right)}{1-r^{\left(i_{j}\right)}},
$$

where

$$
\pi(\varnothing)=\prod_{j=1}^{M}\left(1-r^{(j)}\right)
$$
by

In the p.g.fl. form, the distribution function in (4) is given

$$
G[h]=\prod_{i=1}^{M}\left(1-r^{(i)}+r^{(i)}\left\langle p^{(i)}, h\right\rangle\right) .
$$

2.2. MeMBer and CBMeMBer Filters. In the MeMBer filter, the distribution of a multi-Bernoulli RFS is parameterized by existence probabilities and probability densities of hypothesized states. Given a parametric posterior distribution $\left\{\left(r_{k-1}^{(i)}, p_{k-1}^{(i)}\right)\right\}_{i=1}^{M_{k-1}}$ at time $k-1$, the MeMBer filter is formed according to the following two steps. Further details can be found in $[1,8,9]$.

2.2.1. Prediction. The predicted distribution is still in the multi-Bernoulli form and consists of two independent multiBernoulli sets:

$$
\pi_{k \mid k-1}=\left\{\left(r_{P, k \mid k-1}^{(i)}, p_{P, k \mid k-1}^{(i)}\right)\right\}_{i=1}^{M_{k-1}} \cup\left\{\left(r_{\Gamma, k}^{(l)}, p_{\Gamma, k}^{(l)}\right)\right\}_{l=1}^{M_{\Gamma, k}},
$$

where $\left\{\left(r_{\Gamma, k}^{(l)}, p_{\Gamma, k}^{(l)}\right)\right\}_{l=1}^{M_{\Gamma, k}}$ denotes the multi-Bernoulli set of the newborn targets, and $\left\{\left(r_{P, k \mid k-1}^{(i)}, p_{P, k \mid k-1}^{(i)}\right)\right\}_{i=1}^{M_{k-1}}$ models the surviving targets.

Suppose that each target survives from time $k-1$ with probability $p_{S, k}\left(\boldsymbol{x}_{k-1}\right)$ and the transition density is $f_{k \mid k-1}(\boldsymbol{x} \mid$ $\left.\boldsymbol{x}_{k-1}\right)$; thus the predicted multi-Bernoulli RFS of surviving targets can be described by the following [9]:

$$
\begin{aligned}
r_{P, k \mid k-1}^{(i)} & =r_{k-1}^{(i)}\left\langle p_{k-1}^{(i)}, p_{S, k}\right\rangle, \\
p_{P, k \mid k-1}^{(i)}(\boldsymbol{x}) & =\frac{\left\langle f_{k \mid k-1}(\boldsymbol{x} \mid \bullet), p_{k-1}^{(i)} p_{S, k}\right\rangle}{\left\langle p_{k-1}^{(i)}, p_{S, k}\right\rangle} .
\end{aligned}
$$

2.2.2. Update. The posterior distribution at time $k$ is approximated by the union of the multi-Bernoulli RFSs for legacy tracks and data-induced tracks, that is,

$$
\pi_{k} \approx\left\{\left(r_{L, k}^{(i)}, p_{L, k}^{(i)}\right)\right\}_{i=1}^{M_{k \mid k-1}} \cup\left\{\left(r_{U, k}(\boldsymbol{z}), p_{U, k}(\boldsymbol{x} ; \boldsymbol{z})\right)\right\}_{\boldsymbol{z} \in \boldsymbol{Z}_{k}} .
$$

The parameters in (10) are given by the following [9]:

$$
r_{L, k}^{(i)}=r_{k \mid k-1}^{(i)} \frac{1-\left\langle p_{k \mid k-1}^{(i)}, p_{D, k}\right\rangle}{1-r_{k \mid k-1}^{(i)}\left\langle p_{k \mid k-1}^{(i)}, p_{D, k}\right\rangle},
$$




$$
\begin{aligned}
p_{L, k}^{(i)}(\boldsymbol{x}) & =p_{k \mid k-1}^{(i)}(\boldsymbol{x}) \frac{1-p_{D, k}(\boldsymbol{x})}{1-\left\langle p_{k \mid k-1}^{(i)}, p_{D, k}\right\rangle} . \\
r_{U, k}(\boldsymbol{z}) & =\frac{\sum_{i=1}^{M_{k \mid k-1}}\left(r_{k \mid k-1}^{(i)}\left\langle p_{k \mid k-1}^{(i)}, p_{D, k} g_{k, z}\right\rangle /\left(1-r_{k \mid k-1}^{(i)}\left\langle p_{k \mid k-1}^{(i)}, p_{D, k}\right\rangle\right)\right)}{\kappa_{k}(\boldsymbol{z})+\sum_{i=1}^{M_{k \mid k-1}}\left(r_{k \mid k-1}^{(i)}\left\langle p_{k \mid k-1}^{(i)}, p_{D, k} g_{k, \boldsymbol{z}}\right\rangle /\left(1-r_{k \mid k-1}^{(i)}\left\langle p_{k \mid k-1}^{(i)}, p_{D, k}\right\rangle\right)\right)}, \\
p_{U, k}(\boldsymbol{x} ; \boldsymbol{z}) & =\frac{\sum_{i=1}^{M_{k \mid k-1}}\left(r_{k \mid k-1}^{(i)} p_{k \mid k-1}^{(i)} p_{D, k}(\boldsymbol{x}) g_{k, z}(\boldsymbol{z} \mid \boldsymbol{x}) /\left(1-r_{k \mid k-1}^{(i)}\left\langle p_{k \mid k-1}^{(i)}, p_{D, k}\right\rangle\right)\right)}{\sum_{i=1}^{M_{k \mid k-1}}\left(r_{k \mid k-1}^{(i)}\left\langle p_{k \mid k-1}^{(i)}, p_{D, k} g_{k, \boldsymbol{z}}\right\rangle /\left(1-r_{k \mid k-1}^{(i)}\left\langle p_{k \mid k-1}^{(i)}, p_{D, k}\right\rangle\right)\right)},
\end{aligned}
$$

where $\boldsymbol{Z}_{k}$ denotes the measurement set, $p_{D, k}(\boldsymbol{x})$ denotes the detection probability, $g_{k, z}(\boldsymbol{z} \mid \boldsymbol{x})$ denotes the likelihood function, and $\kappa_{k}(\boldsymbol{z})$ denotes the intensity of Poisson clutter at time $k$.

In order to get (13) and (14), the MeMBer filter makes an approximation in the derivation process which leads to the cardinality bias. This bias is removed in the CBMeMBer filter because the existence probabilities of targets are calculated exactly. The CBMeMBer filter differs from the original MeMBer filter in the multi-Bernoulli RFS set for data-induced tracks, that is [8],

$$
\begin{aligned}
r_{U, k}^{*}(z) & =\frac{\sum_{i=1}^{M_{k \mid k-1}}\left(r_{k \mid k-1}^{(i)}\left(1-r_{k \mid k-1}^{(i)}\right)\left\langle p_{k \mid k-1}^{(i)}, p_{D, k} g_{k, z}\right\rangle /\left(1-r_{k \mid k-1}^{(i)}\left\langle p_{k \mid k-1}^{(i)}, p_{D, k}\right\rangle\right)^{2}\right)}{\kappa_{k}(\boldsymbol{z})+\sum_{i=1}^{M_{k \mid k-1}}\left(r_{k \mid k-1}^{(i)}\left\langle p_{k \mid k-1}^{(i)}, p_{D, k} g_{k, z}\right\rangle /\left(1-r_{k \mid k-1}^{(i)}\left\langle p_{k \mid k-1}^{(i)}, p_{D, k}\right\rangle\right)\right)}, \\
p_{U, k}^{*}(\boldsymbol{x} ; \boldsymbol{z}) & =\frac{\sum_{i=1}^{M_{k \mid k-1}}\left(\left(r_{k \mid k-1}^{(i)} /\left(1-r_{k \mid k-1}^{(i)}\right)\right) p_{k \mid k-1}^{(i)} p_{D, k}(\boldsymbol{x}) g_{k, \boldsymbol{z}}(\boldsymbol{z} \mid \boldsymbol{x})\right)}{\sum_{i=1}^{M_{k \mid k-1}}\left(\left(r_{k \mid k-1}^{(i)} /\left(1-r_{k \mid k-1}^{(i)}\right)\right)\left\langle p_{k \mid k-1}^{(i)}, p_{D, k} g_{k, z}\right\rangle\right)}
\end{aligned}
$$

\section{Measurement-Driven MeMBer Filter}

3.1. Problem Formulation. While the prediction step of the MeMBer filter is accurate, the approximation made in the update step gives rise to the cardinality bias. Assume that the clutter is sparse, the p.g.fl. of the updated distribution can be approximated by the following [1]:

$$
G_{k}[h] \approx \prod_{i=1}^{M_{k \mid k-1}} G_{L, k}^{(i)}[h] \prod_{z \in Z_{k}} G_{U, k}[z ; h],
$$

where

$$
\begin{aligned}
G_{L, k}^{(i)}[h] & =\frac{1-r_{k \mid k-1}^{(i)}+r_{k \mid k-1}^{(i)}\left\langle p_{k \mid k-1}^{(i)}, h q_{D, k}\right\rangle}{1-r_{k \mid k-1}^{(i)}+r_{k \mid k-1}^{(i)}\left\langle p_{k \mid k-1}^{(i)}, q_{D, k}\right\rangle}, \\
G_{U, k}[z ; h] & =\frac{\kappa_{k}(z)+\sum_{i=1}^{M_{k \mid k-1}} G_{U, k}^{(i)}[z ; h]}{\kappa_{k}(z)+\sum_{i=1}^{M_{k \mid k-1}} G_{U, k}^{(i)}[z ; 1]}, \\
G_{U, k}^{(i)}[z ; h] & =\frac{r_{k \mid k-1}^{(i)}\left\langle p_{k \mid k-1}^{(i)}, h p_{D, k} g_{k, z}\right\rangle}{1-r_{k \mid k-1}^{(i)}+r_{k \mid k-1}^{(i)}\left\langle p_{k \mid k-1}^{(i)}, h q_{D, k}\right\rangle}, \\
q_{D, k} & =1-p_{D, k} .
\end{aligned}
$$

The first product in (17) which corresponds to the updates of assumed undetected targets is in the form of multiBernoulli, while the second product which corresponds to the updates of all the predicted targets is not of multi-Bernoulli form. To maintain the recursion of the MeMBer filter, Mahler set $h=1$ in the denominator of $G_{U, k}^{(i)}[z ; h][1]$, that is,

$$
\begin{aligned}
G_{U, k}^{(i)}[z ; h] & \approx \frac{r_{k \mid k-1}^{(i)}\left\langle p_{k \mid k-1}^{(i)}, h p_{D, k} g_{k, z}\right\rangle}{1-r_{k \mid k-1}^{(i)}+r_{k \mid k-1}^{(i)}\left\langle p_{k \mid k-1}^{(i)}, q_{D, k}\right\rangle} \\
& =\frac{r_{k \mid k-1}^{(i)}\left\langle p_{k \mid k-1}^{(i)}, h p_{D, k} g_{k, z}\right\rangle}{1-r_{k \mid k-1}^{(i)}\left\langle p_{k \mid k-1}^{(i)}, p_{D, k}\right\rangle} .
\end{aligned}
$$

This approximation ensures that the second product in (17) can also be written in the multi-Bernoulli form; however, it also leads to the fact that the existence probabilities calculated from (22) are not accurate enough which introduces the cardinality bias. Though the CBMeMBer filter removes the bias by calculating the exact existence probabilities instead, both of these two filters somewhat reduce the contribution that the measurements make to the posterior distribution. To fully use the measurements information, the probabilities of measurements originating from targets are deduced as follows and will be further used to refine the update step. Given measurement $z$, it originates from either the targets with probability density $\sum_{i=1}^{M_{k \mid k-1}} r_{k \mid k-1}^{(i)}\left\langle p_{k \mid k-1}^{(i)}, p_{D, k} g_{k, z}\right\rangle$ or the clutter with probability density $\kappa_{k}(\boldsymbol{z})$; thus the probability of measurement $\boldsymbol{z}$ originating from target $\boldsymbol{x}_{i}$ can be computed as 


$$
R_{z}^{(i)} \approx \frac{r_{k \mid k-1}^{(i)}\left\langle p_{k \mid k-1}^{(i)}, \psi_{k, z}\right\rangle}{\kappa_{k}(z)+\sum_{i=1}^{M_{k \mid k-1}} r_{k \mid k-1}^{(i)}\left\langle p_{k \mid k-1}^{(i)}, \psi_{k, z}\right\rangle} .
$$

Given all the measurements, the probability of measurements originating from target $\boldsymbol{x}_{i}$ can be expressed as a union of all the individual corresponding probabilities, that is,

$$
\begin{aligned}
R^{(i)} & =\sum_{\boldsymbol{z} \in \boldsymbol{Z}_{k}} R_{z}^{(i)} \\
& \approx \sum_{\boldsymbol{z} \in \boldsymbol{Z}_{k}} \frac{r_{k \mid k-1}^{(i)}\left\langle p_{k \mid k-1}^{(i)}, \psi_{k, z}\right\rangle}{\kappa_{k}(\boldsymbol{z})+\sum_{i=1}^{M_{k \mid k-1}} r_{k \mid k-1}^{(i)}\left\langle p_{k \mid k-1}^{(i)}, \psi_{k, z}\right\rangle} .
\end{aligned}
$$

It is reasonable that $R^{(i)}$ has a significant impact on the value of $r_{L, k}^{(i)}$, because $r_{L, k}^{(i)}$ denotes the existence probabilities of the assumed undetected target and the measurements contain the information about detection; however, there is no measurement information contained in the expression of $r_{L, k}^{(i)}$. To solve this problem, while the prediction step of the original MeMBer filter is accurate [9], the refinement of the updates under legacy track set and date-induced track set is given as follows.

3.2. Refinement of Updates under Legacy Track Set. Because of the detection information contained in $R^{(i)}$, it is further used to refine the updates of legacy tracks which correspond to undetected targets. For each predicted target, when the probability $R^{(i)}$ is given, the corresponding p.g.fl. $G_{L, k}^{(i)}[h]$ can be divided into two terms, that is,

$$
G_{L, k}^{(i)}[h]=\left(1-R^{(i)}\right) G_{L, k}^{(i)}[h]+R^{(i)} G_{L, k}^{(i)}[h],
$$

where the first term corresponds to the refined update of the target under legacy track set, and it is used in the following to deduce the expressions of modified existence probability and probability density, while the second term corresponds to part of the cardinality bias in the MeMBer filter, the other part of the cardinality bias will be represented in the next section.

According to (18) and (25), the refined p.g.fl. of updated distribution under legacy track set can be expressed as

$$
\begin{aligned}
G_{L, k}^{\circ(i)} & {[h]=\left(1-R^{(i)}\right) G_{L, k}^{(i)}[h] } \\
= & \left(1-R^{(i)}\right) \frac{1-r_{k \mid k-1}^{(i)}+r_{k \mid k-1}^{(i)}\left\langle p_{k \mid k-1}^{(i)}, h q_{D, k}\right\rangle}{1-r_{k \mid k-1}^{(i)}+r_{k \mid k-1}^{(i)}\left\langle p_{k \mid k-1}^{(i)}, q_{D, k}\right\rangle},
\end{aligned}
$$

Let parameter pair $\left(r_{L, k}^{\circ(i)}, p_{L, k}^{\circ(i)}\right)$ denote the modified existence probability and probability density; the expression of the parameters can be deduced as follows. Set $h=y$, the probability generating function of $G_{L, k}^{\circ(i)}[h]$ is represented as $G_{L, k}^{\circ(i)}(y)$, and the exact value of $r_{L, k}^{\circ(i)}$ can be calculated from the differential of $G_{L, k}^{\circ(i)}(y)$ at $y=1$, that is

$$
\begin{aligned}
r_{L, k}^{\circ(i)} & =G_{L, k}^{\circ(i)^{\prime}}(1) \\
& =\left(1-R^{(i)}\right) r_{k \mid k-1}^{(i)} \frac{1-\left\langle p_{k \mid k-1}^{(i)}, p_{D, k}\right\rangle}{1-r_{k \mid k-1}^{(i)}\left\langle p_{k \mid k-1}^{(i)}, p_{D, k}\right\rangle} .
\end{aligned}
$$

To obtain the expression of $p_{L, k}^{\circ(i)}$, the intensity function $r_{L, k}^{\circ(i)} p_{L, k}^{\circ(i)}$ is computed first by taking the Frechét derivative of $G_{L, k}^{\circ(i)}[h]$ at $h=1$, that is

$$
\begin{aligned}
r_{L, k}^{\circ(i)} p_{L, k}^{\circ(i)} & =\frac{\delta G_{L, k}^{\circ(i)}}{\delta \boldsymbol{x}}[1]=\lim _{\varepsilon \searrow 0} \frac{G_{L, k}^{\circ(i)}\left[1+\varepsilon \delta_{x}\right]-G_{L, k}^{\circ(i)}[1]}{\varepsilon} \\
& =\left(1-R^{(i)}\right) \frac{r_{k \mid k-1}^{(i)}\left(1-p_{D, k}\right)}{1-r_{k \mid k-1}^{(i)}\left\langle p_{k \mid k-1}^{(i)}, p_{D, k}\right\rangle} p_{k \mid k-1}^{(i)} .
\end{aligned}
$$

Dividing the intensity function in (28) by $(27), p_{L, k}^{\circ(i)}$ is given by

$$
p_{L, k}^{\circ(i)}=\frac{\left(1-p_{D, k}\right)}{1-\left\langle p_{k \mid k-1}^{(i)}, p_{D, k}\right\rangle} p_{k \mid k-1}^{(i)} .
$$

Equations (27) and (29) constitute the refined updates of targets under legacy track set; compared with (11) and (12), the existence probabilities are multiplied by $\left(1-R^{(i)}\right)$, while the probability densities have the same form.

3.3. Refinement of Updates under Data-Induced Track Set. The updates under legacy track set and data-induced track set correspond to the assumed undetected targets and the assumed detected targets, respectively. For each target, since the update under legacy track set have been modified by the measurements in the previous section, the update under data-induced track set should also be modified, because they correspond to one same target and different models for one target lead to poor performance. If a target produces the measurement with a low probability, the contribution of the measurement to the update under data-induced track set will decrease.

Considering the analysis above, the data-induced updates are further refined as follows. Suppose that $G_{L, k}^{(i)}[h]$ and $G_{U, k}^{(i)}[z ; h]$ correspond to the same target; unlike $G_{L, k}^{(i)}[h]$, $G_{U, k}^{(i)}[z ; h]$ already contains the measurements information; thus the existence probabilities of the targets under legacy track set are directly used to modify $G_{U, k}^{(i)}[z ; h]$ :

$$
G_{U, k}^{(i)}[z ; h]=\left(1-r_{L, k}^{\circ(i)}\right) G_{U, k}^{(i)}[z ; h]+r_{L, k}^{\circ(i)} G_{U, k}^{(i)}[z ; h],
$$

where the first term corresponds to the refined data-induced update, while the second term represents part of the cardinality bias in the MeMBer filter, and the rest of the bias has been demonstrated in (25).

Let $G_{U, k}^{\circ}[z ; h]$ be denoted as the refined p.g.fl. of the updated distribution under data-induced track set; according to (19) and (30), the expression of $G_{U, k}^{\circ}[z ; h]$ is given by

$$
\begin{aligned}
& G_{U, k}^{\circ}[z ; h]=\frac{\kappa_{k}(z)+\sum_{i=1}^{M_{k \mid k-1}} G_{U, k}^{\circ(i)}[z ; h]}{\kappa_{k}(z)+\sum_{i=1}^{M_{k \mid k-1}} G_{U, k}^{(i)}[z ; 1]}, \\
& G_{U, k}^{\circ(i)}[z ; h]=\left(1-r_{L, k}^{\circ(i)}\right) G_{U, k}^{(i)}[z ; h],
\end{aligned}
$$

where $G_{U, k}^{(i)}[z ; h]$ is given by $(20)$. 
Let parameter pair $\left(r_{U, k}^{\circ}(\boldsymbol{z}), p_{U, k}^{\circ}(\boldsymbol{z})\right)$ denote the modified existence probability and probability density under datainduced track set; the derivation processes of $r_{U, k}^{\circ}(z)$ and
$p_{U, k}^{\circ}(\boldsymbol{z})$ are similar to those of $r_{L, k}^{\circ(i)}$ and $p_{L, k}^{\circ(i)}$. Thus $r_{U, k}^{\circ}(\boldsymbol{z})$ is given by

$$
r_{U, k}^{\circ}(z)=G_{U, k}^{\circ}{ }^{\prime}(z ; 1)=\frac{\sum_{i=1}^{M_{k \mid k-1}}\left(\left(1-r_{L, k}^{\circ(i)}\right) r_{k \mid k-1}^{(i)}\left\langle p_{k \mid k-1}^{(i)}, p_{D, k} g_{k, z}\right\rangle /\left(1-r_{k \mid k-1}^{(i)}\left\langle p_{k \mid k-1}^{(i)}, p_{D, k}\right\rangle\right)\right)}{\kappa_{k}(z)+\sum_{i=1}^{M_{k \mid k-1}}\left(r_{k \mid k-1}^{(i)}\left\langle p_{k \mid k-1}^{(i)}, p_{D, k} g_{k, z}\right\rangle /\left(1-r_{k \mid k-1}^{(i)}\left\langle p_{k \mid k-1}^{(i)}, p_{D, k}\right\rangle\right)\right)} .
$$

The intensity function is computed as

$$
\begin{aligned}
r_{U, k}^{\circ}(\boldsymbol{z}) p_{U, k}^{\circ}(\boldsymbol{z}) & =\lim _{\varepsilon \searrow 0} \frac{G_{U, k}^{\circ}\left[\boldsymbol{z} ; 1+\varepsilon \delta_{\boldsymbol{x}}\right]-G_{L, k}^{\circ(i)}[\boldsymbol{z} ; 1]}{\varepsilon} \\
& =\frac{\sum_{i=1}^{M_{k \mid k-1}}\left(\left(1-r_{L, k}^{\circ(i)}\right) r_{k \mid k-1}^{(i)} p_{k \mid k-1}^{(i)} p_{D, k} g_{k, z} /\left(1-r_{k \mid k-1}^{(i)}\left\langle p_{k \mid k-1}^{(i)}, p_{D, k}\right\rangle\right)\right)}{\kappa_{k}(\boldsymbol{z})+\sum_{i=1}^{M_{k \mid k-1}}\left(r_{k \mid k-1}^{(i)}\left\langle p_{k \mid k-1}^{(i)}, p_{D, k} g_{k, z}\right\rangle /\left(1-r_{k \mid k-1}^{(i)}\left\langle p_{k \mid k-1}^{(i)}, p_{D, k}\right\rangle\right)\right)} .
\end{aligned}
$$

And $p_{U, k}^{\circ}(\boldsymbol{z})$ is given by dividing (34) by (33):

$$
p_{U, k}^{\circ}(z)=\frac{\sum_{i=1}^{M_{k \mid k-1}}\left(\left(1-r_{L, k}^{\circ(i)}\right) r_{k \mid k-1}^{(i)} p_{k \mid k-1}^{(i)} p_{D, k} g_{k, z} /\left(1-r_{k \mid k-1}^{(i)}\left\langle p_{k \mid k-1}^{(i)}, p_{D, k}\right\rangle\right)\right)}{\sum_{i=1}^{M_{k \mid k-1}}\left(\left(1-r_{L, k}^{\circ(i)}\right) r_{k \mid k-1}^{(i)}\left\langle p_{k \mid k-1}^{(i)}, p_{D, k} g_{k, z}\right\rangle /\left(1-r_{k \mid k-1}^{(i)}\left\langle p_{k \mid k-1}^{(i)}, p_{D, k}\right\rangle\right)\right)} .
$$

Equations (27), (29), (33), and (35) constitute the refined update step of the proposed filter; compared with this filter, the CBMeMBer filter reduces the measurements' contribution to the update step and makes the approximation $p_{D, k} \approx 1$ to obtain a valid $p_{U, k}^{*}(\boldsymbol{x} ; \boldsymbol{z})$. Observe that there is no approximation on $p_{D, k}$ in the proposed measurementdriven filter, the proposed filter removes the high signal-tonoise ratio assumption. However, they both assume that the clutter is not dense in order to obtain the approximation in (17).

\section{Simulations and Discussions}

In this section, the performance of the proposed measurement-driven multi-target multi-Bernoulli filter is validated via the following example shown in Figure 1. There are at most ten targets that appear or disappear on this scene, and the births and deaths of targets occur at various time points. The performance of the proposed filter is compared with the CBMeMBer and IMeMBer filters through the Gaussian mixture implementation.

Let $\boldsymbol{x}_{k}=\left[x_{k}, y_{k}, \dot{x}_{k}, \dot{y}_{k}, \omega_{k}\right]^{T}$ denote the target state variable with position $\left[x_{k}, y_{k}\right]$, velocity $\left[\dot{x}_{k}, \dot{y}_{k}\right]$, and turn rate $\omega_{k}$; the dynamic equation is given by

$$
\boldsymbol{x}_{k}=\boldsymbol{F}\left(\omega_{k-1}\right) \boldsymbol{x}_{k-1}+\boldsymbol{\Gamma} \boldsymbol{w}_{k-1},
$$

where

$$
\begin{aligned}
& \boldsymbol{F}\left(\omega_{k-1}\right) \\
& =\left[\begin{array}{ccccc}
1 & 0 & \frac{\sin \left(\omega_{k-1} \Delta t\right)}{\omega_{k-1}} & \frac{\cos \left(\omega_{k-1} \Delta t\right)-1}{\omega_{k-1}} & 0 \\
0 & 1 & \frac{1-\cos \left(\omega_{k-1} \Delta t\right)}{\omega_{k-1}} & \frac{\sin \left(\omega_{k-1} \Delta t\right)}{\omega_{k-1}} & 0 \\
0 & 0 & \cos \left(\omega_{k-1} \Delta t\right) & -\sin \left(\omega_{k-1} \Delta t\right) & 0 \\
0 & 0 & \sin \left(\omega_{k-1} \Delta t\right) & \cos \left(\omega_{k-1} \Delta t\right) & 0 \\
0 & 0 & 0 & 0 & 1
\end{array}\right] \text {, } \\
& \Gamma=\left[\begin{array}{ccc}
\frac{\Delta t^{2}}{2} & 0 & 0 \\
0 & \frac{\Delta t^{2}}{2} & 0 \\
\Delta t & 0 & 0 \\
0 & \Delta t & 0 \\
0 & 0 & \Delta t
\end{array}\right]
\end{aligned}
$$

$\boldsymbol{w}_{k-1}=\left[\begin{array}{ll}\widetilde{\boldsymbol{w}}_{k-1}^{T} & u_{k-1}\end{array}\right]^{T}, \widetilde{\boldsymbol{w}}_{k-1}^{T} \sim N\left(\cdot ; 0, \sigma_{\widetilde{\boldsymbol{w}}}^{2} \boldsymbol{I}\right)$ is the process noise, $u_{k-1} \sim N\left(\cdot ; 0, \sigma_{u}^{2} \boldsymbol{I}\right)$ is the turn rate noise, $\sigma_{\widetilde{\boldsymbol{w}}}=15 \mathrm{~m} / \mathrm{s}^{2}$, $\sigma_{u}=\pi / 180 \mathrm{rad} / \mathrm{s}, \Delta t=1 \mathrm{~s}$ is the sampling period. The birth process is modeled by a multi-Bernoulli set $\left\{\left(r_{\Gamma}^{(i)}, p_{\Gamma}^{(i)}\right)\right\}_{i=1}^{4}$, where $r_{\Gamma}^{(1)}=r_{\Gamma}^{(2)}=0.02, r_{\Gamma}^{(3)}=r_{\Gamma}^{(4)}=0.03, p_{\Gamma}^{(i)} \sim N\left(x ; m_{\Gamma}^{(i)}, P_{\Gamma}\right)$, 


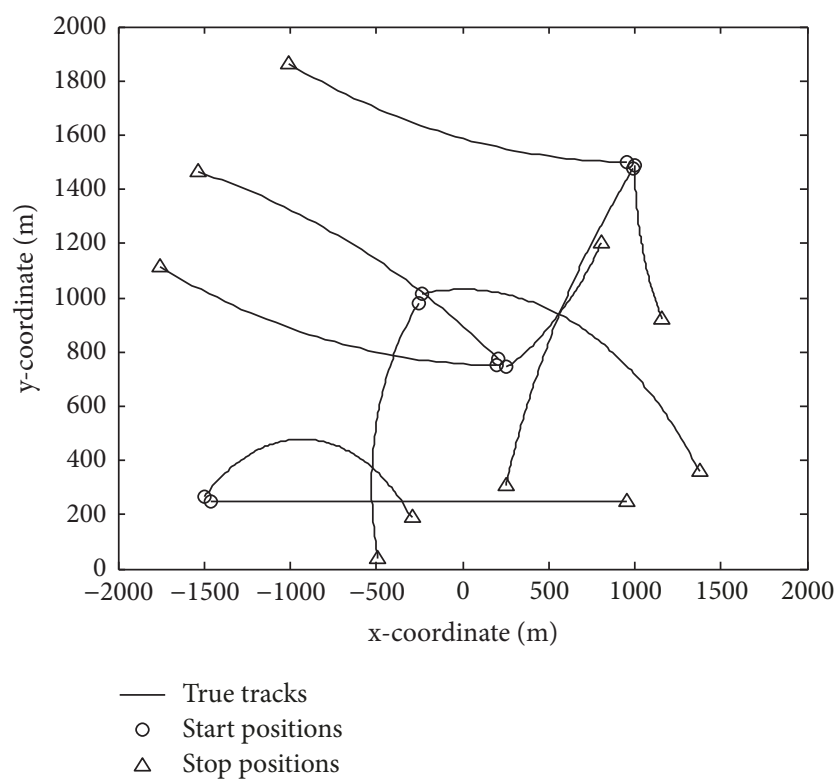

FIGURE 1: True tracks of targets.

$m_{\Gamma}^{(1)}=[-1500,0,250,0,0], m_{\Gamma}^{(2)}=[-250,0,1000,0,0]$, $m_{\Gamma}^{(3)}=[250,0,750,0,0], m_{\Gamma}^{(4)}=[1000,0,1500,0,0], P_{\Gamma}=$ $\operatorname{diag}\left([50,50,50,50,6(\pi / 180)]^{T}\right)^{2}$. The probability of targets surviving from last time point is 0.99 .

Suppose that the targets are observed with missed detection and clutter; when a target is detected, the observation is modeled by

$$
\boldsymbol{z}_{k}=\boldsymbol{H}\left(\boldsymbol{x}_{k}\right)+\varepsilon_{k}
$$

where $\boldsymbol{H}\left(\boldsymbol{x}_{k}\right)=\left[\arctan \left(y_{k} / x_{k}\right), \sqrt{x_{k}^{2}+y_{k}^{2}}\right]^{T}, \varepsilon_{k} \sim N\left(\cdot ; 0, \boldsymbol{R}_{k}\right)$ is the measurement noise, $\boldsymbol{R}_{k}=\operatorname{diag}\left(\left[(\pi / 180 \mathrm{rad})^{2},(5 \mathrm{~m})^{2}\right]\right)$. The clutter is Poisson distributed with an average of 15 points per scan over the region $[0, \pi] \mathrm{rad} \times[0,2000] \mathrm{m}$; that is, the intensity of clutter is $2.4 \times 10^{-3}(\mathrm{radm})^{-1}$. The probability of detection is 0.9 .

For each filter, 500 Monte Carlo runs with same target tracks and randomly generated measurements are performed. Figure 2 shows the $\mathrm{x}$ and $\mathrm{y}$ components of the measurements, true tracks, and state estimates from the proposed filter. The results suggest that the proposed filter is able to track each individual target correctly and identify the births and deaths of various targets throughout the scenario. The crossings of targets are also well handled.

The mean values and standard deviations of the cardinality estimates for different filters are shown versus time in Figures 3 and 4 . The simulation results indicate that the three filters are all unbiased, while, for the stability of cardinality estimation, the proposed filter has the lowest standard deviation, and the IMeMBer filter has the worst performance because it does not modify the existence probabilities under data-induced track set.

The optimal subpattern assignment (OSPA) distance is used to evaluate the performance of different filters. The OSPA distance measures the metric between two RFSs based
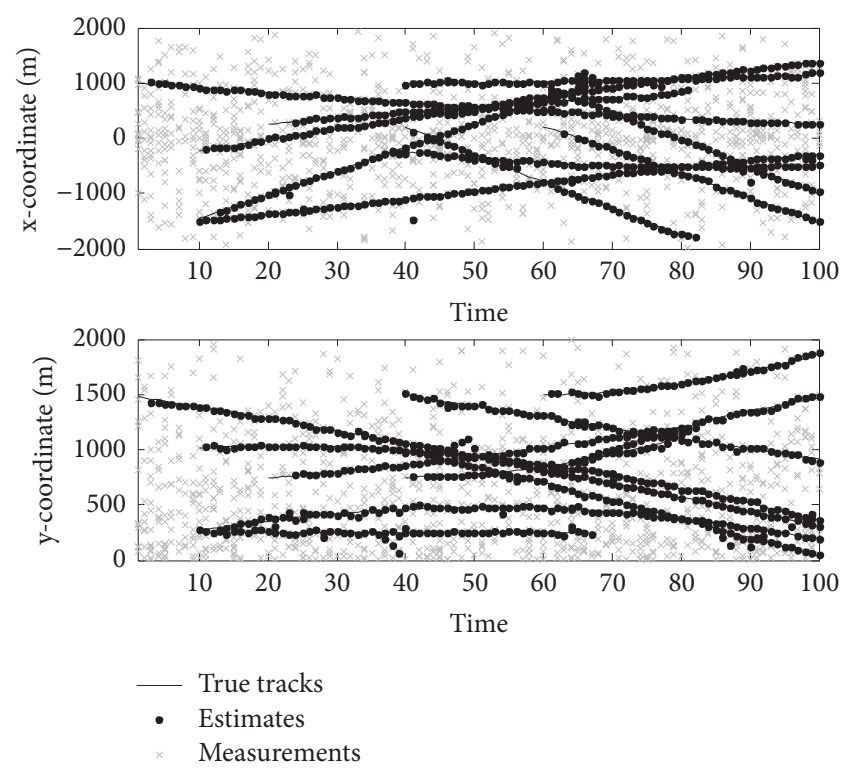

FIGURE 2: Measurements, true tracks, and proposed filter estimates.
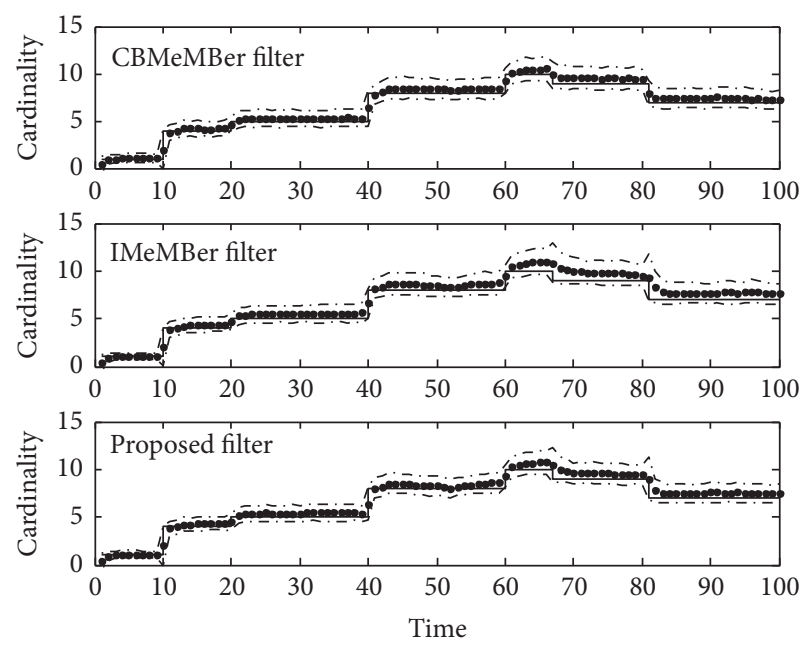

FIgUre 3: Cardinality statistics for the CBMeMBer, IMeMBer, and proposed filters.

on an optimal assignment algorithm, and it consists of two components: cardinality error and localization error. The OSPA distance between $X=\left\{\boldsymbol{x}_{1}, \cdots, \boldsymbol{x}_{m}\right\}$ and $Y=$ $\left\{\boldsymbol{y}_{1}, \cdots, \boldsymbol{y}_{m}\right\}$ can be given by the following [11]:

$$
\begin{aligned}
& \bar{d}_{p}^{(c)}(X, Y) \\
& =\left(\frac{1}{n}\left(\min _{\pi \in \prod_{n}} \sum_{i=1}^{m} d^{(c)}\left(\boldsymbol{x}_{i}, \boldsymbol{y}_{\pi(i)}\right)^{p}+c^{p}(n-m)\right)\right)^{1 / p}
\end{aligned}
$$

where $d^{(c)}(\boldsymbol{x}, \boldsymbol{y})=\min (c,\|\boldsymbol{x}-\boldsymbol{y}\|), \prod_{n}$ denotes the permutations between $X$ and $Y$, the values of $c$ and $p$ determine the 


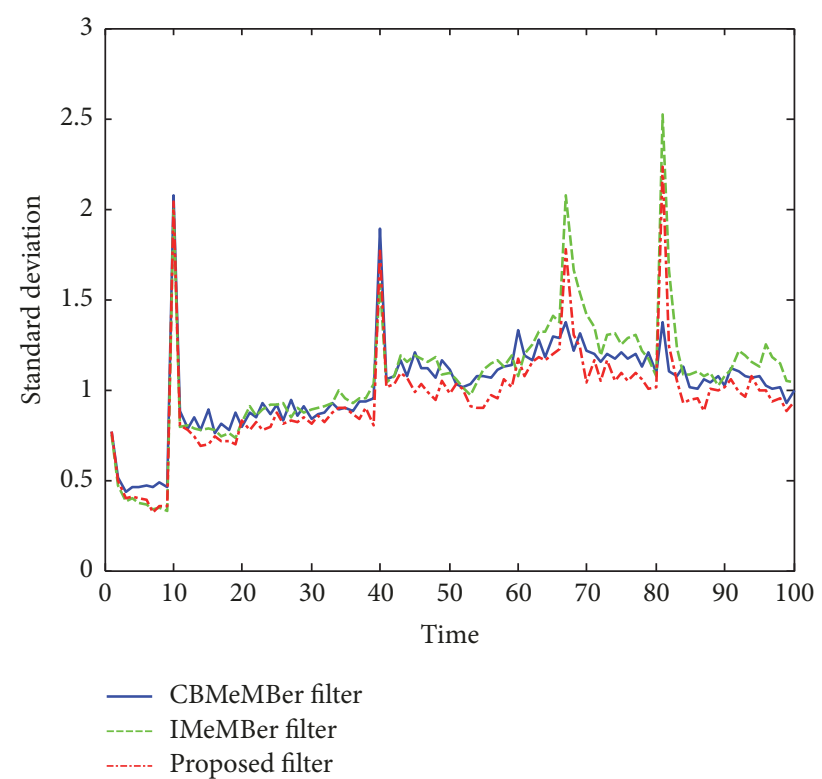

Figure 4: Standard deviations for the CBMeMBer, IMeMBer, and proposed filters.

contribution of cardinality error and localization error to the OSPA distance, respectively. The localization error is given by

$$
\bar{e}_{p, l o c}^{(c)}(X, Y)=\left(\frac{1}{n \pi \in \prod_{n}} \sum_{i=1}^{m} d^{(c)}\left(\boldsymbol{x}_{i}, \boldsymbol{y}_{\pi(i)}\right)^{p}\right)^{1 / p},
$$

while the cardinality error is given by

$$
\bar{e}_{p, \text { card }}^{(c)}(X, Y)=\left(\frac{c^{p}(n-m)}{n}\right)^{1 / p} .
$$

Set $c=1, p=100$; Figure 5 shows the OSPA distances for the three filters. We can see that the proposed filter outperforms the IMeMBer filter, and the IMeMBer filter outperforms the CBMeMBer filter.

Figure 6 shows the OSPA cardinality errors versus time for different filters. It can be seen that the proposed filter performs better than the other two filters for most of the time, and the CBMeMBer filter outperforms the IMeMBer filter. This is due to the mentioned fact that the IMeMBer filter produces the cardinalities under legacy tracks and datainduced tracks with different models which yields the poor performance, while the proposed filter refines the updates under both legacy and data-induced tracks with measurements information.

Figure 7 shows the OSPA localization errors versus time for different filters. The results indicate that the localization estimation performance of the IMeMBer filter is significantly better than that of the CBMeMBer filter; since Figure 6 shows that the differences between cardinality estimation for these two filters are relatively insignificant, it is reasonable that the OSPA distances for the IMeMBer filter are lower than those for the CBMeMBer filter which can be seen in Figure 5, while, for the measurement-driven filter, all the simulation results

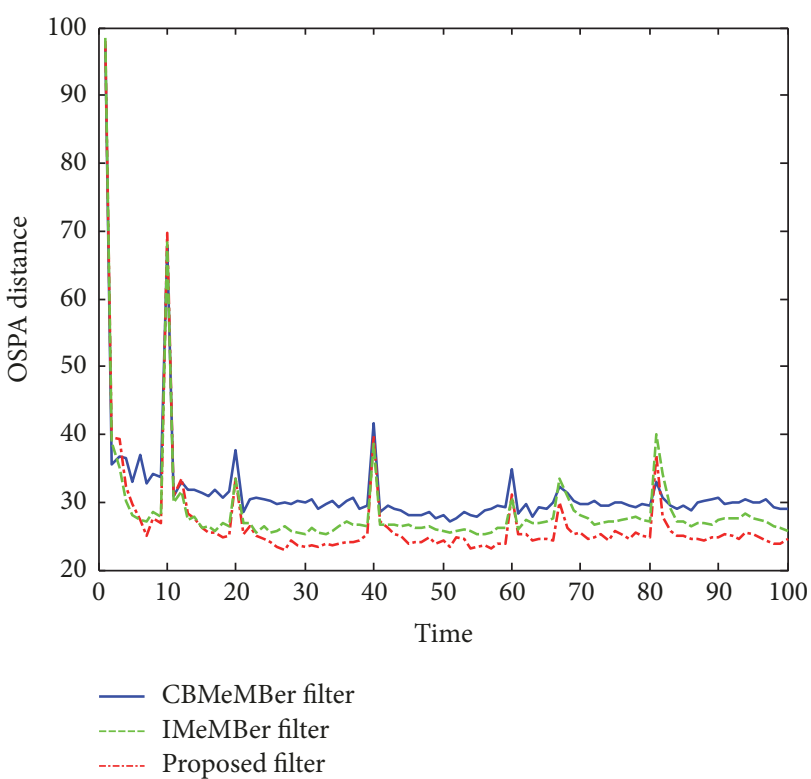

FIGURE 5: OSPA distances for the CBMeMBer, IMeMBer, and proposed filters.

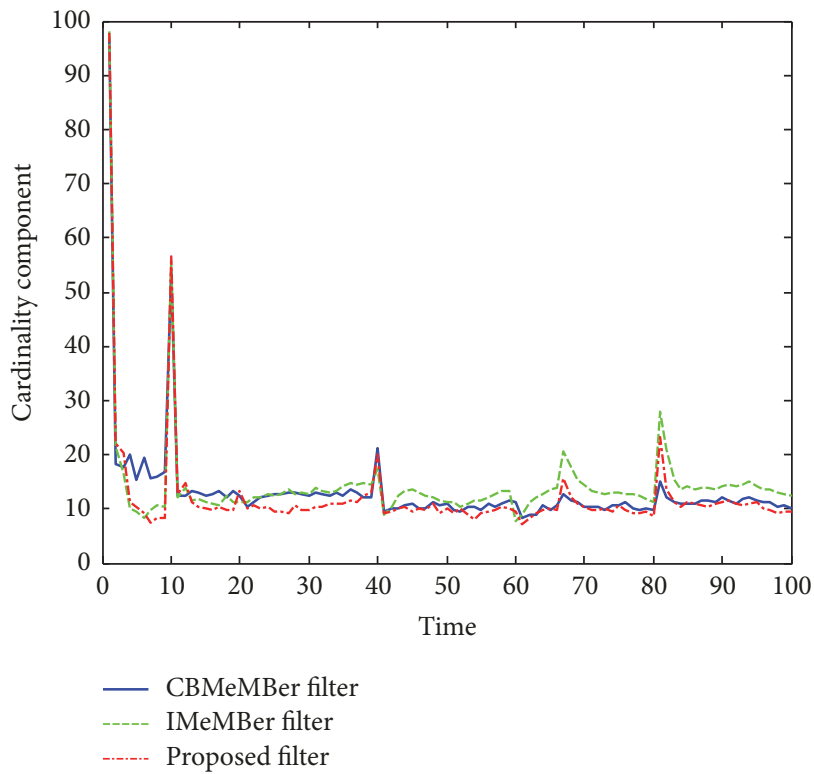

FIGURE 6: OSPA cardinality errors for the CBMeMBer, IMeMBer, and proposed filters.

above show that the proposed filter outperforms the other two filters in both localization and cardinality estimation.

Figure 8 shows the time-averaged OSPA distances for different filters with different detection probabilities. As expected, the proposed filter outperforms the other two filters, and the IMeMBer filter outperforms the CBMeMBer filter. The performance of the CBMeMBer filter gets worse faster with the decrease of detection probability than the other two filters. The results are due to the fact that the CBMeMBer filter assumes $p_{D, k} \approx 1$ while the other two filters make no such assumption on $p_{D, k}$. 


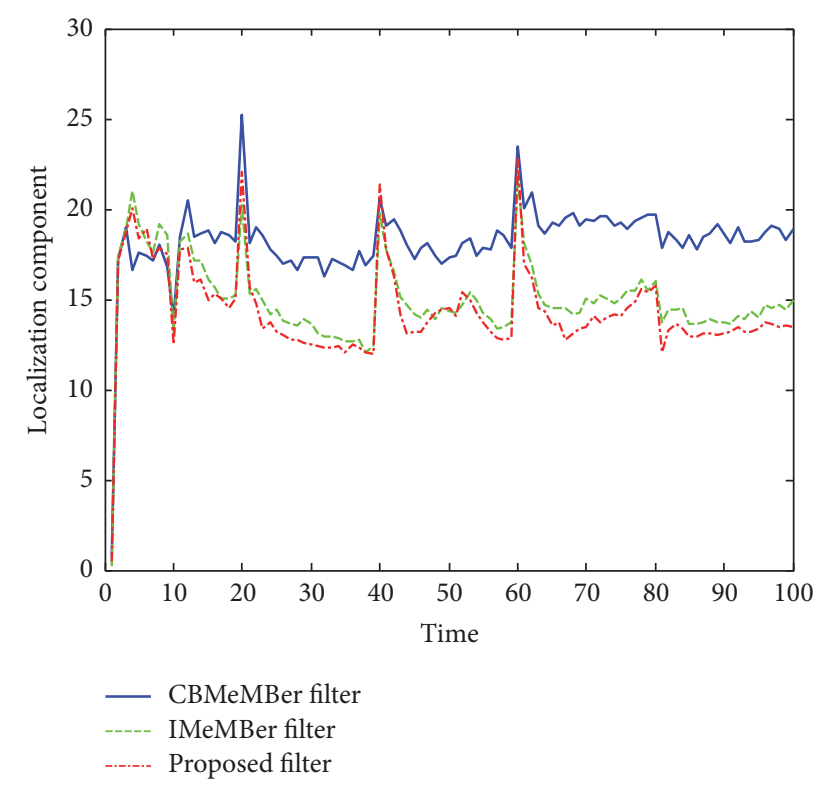

Figure 7: OSPA localization errors for the CBMeMBer, IMeMBer, and proposed filters.

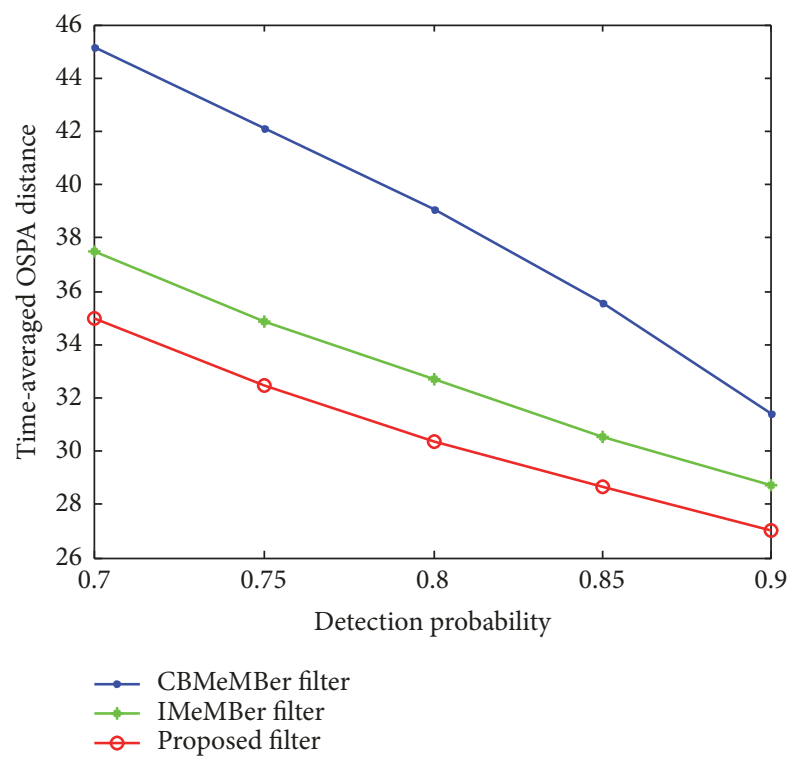

Figure 8: Time-averaged OSPA distances for the CBMeMBer, IMeMBer, and proposed filters.

\section{Conclusions}

The MeMBer filter is positively biased in cardinality because of the approximation made in the update step. To remove this bias, the CBMeMBer filter utilizes the exact corresponding p.g.fl. to calculate the data-induced cardinality estimates. However, both these two filters give insufficient consideration to the measurements. In this paper, a measurement-driven MeMBer filter is proposed, compared with the MeMBer and CBMeMBer filters, the proposed filter uses the measurements information to refine both legacy and date-induced estimates which makes the filter more stable and accurate. The main work of the paper can be summarized as follows.

(1) To refine the updates under legacy track set, the probabilities of measurements originating from targets are deduced and used to refine the p.g.fl. of updated distribution under legacy track set. The modified legacy estimates of existence probabilities and probability densities are further deduced from the refined p.g.fl..

(2) To refine the updates under data-induced track set, the modified existence probabilities of legacy tracks are directly used to improve the multi-target distribution of data-induced tracks.

(3) Simulations are made to compare the performance of the CBMeMBer, IMeMBer, and measurement-driven MeMBer filters. The results show that the proposed filter has more accurate and more stable localization and cardinality estimation, which validates the effectiveness of the proposed filter.

\section{Data Availability}

All the data supporting the conclusions of the study can be found in this manuscript. No additional data are available.

\section{Conflicts of Interest}

The authors declare that they have no conflicts of interest.

\section{Acknowledgments}

This study was supported by the National Natural Science Foundation of China, Grants nos. 61573374, 61503408, 61703421 , and 61773398.

\section{References}

[1] R. Mahler, Statistical Multisource-Multitarget Information Fusion, Artech House, Massachusetts, Mass, USA, 2007.

[2] R. P. S. Mahler, "Multitarget bayes filtering via first-order multitarget moments," IEEE Transactions on Aerospace and Electronic Systems, vol. 39, no. 4, pp. 1152-1178, 2003.

[3] R. Mahler, "PHD filters of higher order in target number," IEEE Transactions on Aerospace and Electronic Systems, vol. 43, no. 4, pp. 1523-1543, 2007.

[4] I. Schlangen, E. D. Delande, J. Houssineau, and D. E. Clark, "A second-order PHD filter with mean and variance in target number," IEEE Transactions on Signal Processing, vol. 66, no. 1, pp. 48-63, 2018.

[5] S. Nagappa, E. D. Delande, D. E. Clark, and J. Houssineau, "A tractable forward-backward CPHD smoother," IEEE Transactions on Aerospace and Electronic Systems, vol. 53, no. 1, pp. 201217, 2017.

[6] N. Daryasafar, R. A. Sadeghzadeh, and M. Naser-Moghadasi, "A technique for multitarget tracking in synthetic aperture radar spotlight imaging mode based on promoted PHD filtering approach," Radio Science, vol. 52, no. 2, pp. 248-258, 2017.

[7] S. Nannuru, S. Blouin, M. Coates, and M. Rabbat, "Multisensor CPHD filter," IEEE Transactions on Aerospace and Electronic Systems, vol. 52, no. 4, pp. 1834-1854, 2016. 
[8] B.-T. Vo, B.-N. Vo, and A. Cantoni, "The cardinality balanced multi-target multi-Bernoulli filter and its implementations," IEEE Transactions on Signal Processing, vol. 57, no. 2, pp. 409423, 2009.

[9] E. Baser, T. Kirubarajan, M. Efe, and B. Balaji, "Improved multitarget multi-Bernoulli filter with modelling of spurious targets," IET Radar, Sonar \& Navigation, vol. 10, no. 2, pp. 285-298, 2016.

[10] B.-T. Vo, B.-N. Vo, R. Hoseinnezhad, and R. P. S. Mahler, "Robust multi-bernoulli filtering," IEEE Journal of Selected Topics in Signal Processing, vol. 7, no. 3, pp. 399-409, 2013.

[11] D. Li, C. Hou, and D. Yi, "Multi-Bernoulli smoother for multitarget tracking," Aerospace Science and Technology, vol. 48, pp. 234-245, 2016

[12] A.-A. Saucan, M. J. Coates, and M. Rabbat, "A multisensor multi-Bernoulli filter," IEEE Transactions on Signal Processing, vol. 65, no. 20, pp. 5495-5509, 2017.

[13] B. Ristic, D. Angley, S. Suvorova et al., "Gaussian mixture multitarget-multisensor Bernoulli tracker for multistatic sonobuoy fields," IET Radar, Sonar and Navigation, vol. 11, no. 12, pp. 1790-1797, 2017.

[14] P. Zong and M. Barbary, "Improved multi-bernoulli filter for extended stealth targets tracking based on sub-random matrices," IEEE Sensors Journal, vol. 16, no. 5, pp. 1428-1447, 2016.

[15] D. Ma, F. Lian, and J. Liu, "Sequential Monte Carlo implementation of cardinality balanced multi-target multi-Bernoulli filter for extended target tracking," IET Radar, Sonar \& Navigation, vol. 10, no. 2, pp. 272-277, 2016.

[16] Y. Qin, H. Ma, L. Cheng, and Y. Li, "Cardinality balanced multitarget multi-Bernoulli filter for multipath multitarget tracking in over-the-horizon radar," IET Radar, Sonar and Navigation, vol. 10, no. 3, pp. 535-545, 2016.

[17] D. Kangin and G. Markarian, "Multi-Bernoulli filter for group object tracking and its Gaussian-Wishart implementation," in Proceedings of the International Joint Conference on Neural Networks, IJCNN '17, Anchorage, Alaska, USA, May 2017.

[18] C. Ouyang, H. Ji, and C. Li, "Improved multi-target multiBernoulli filter," IET Radar, Sonar \& Navigation, vol. 6, no. 6, pp. 458-464, 2012. 


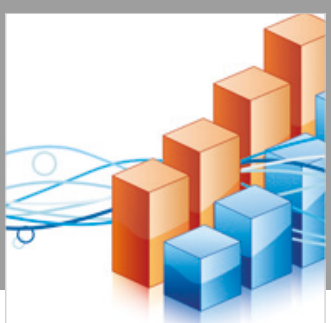

Advances in

Operations Research

\section{-n-m}
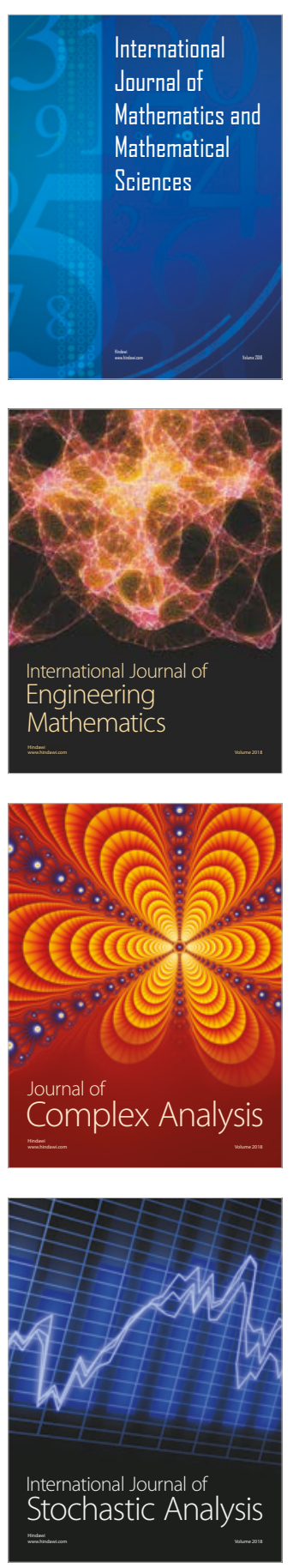
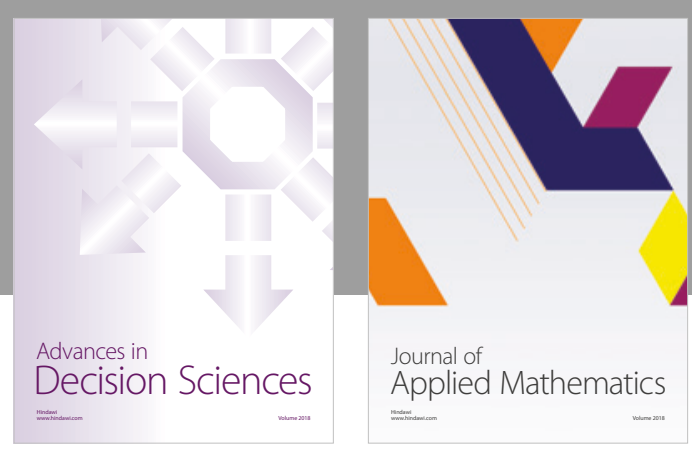

Journal of

Applied Mathematics
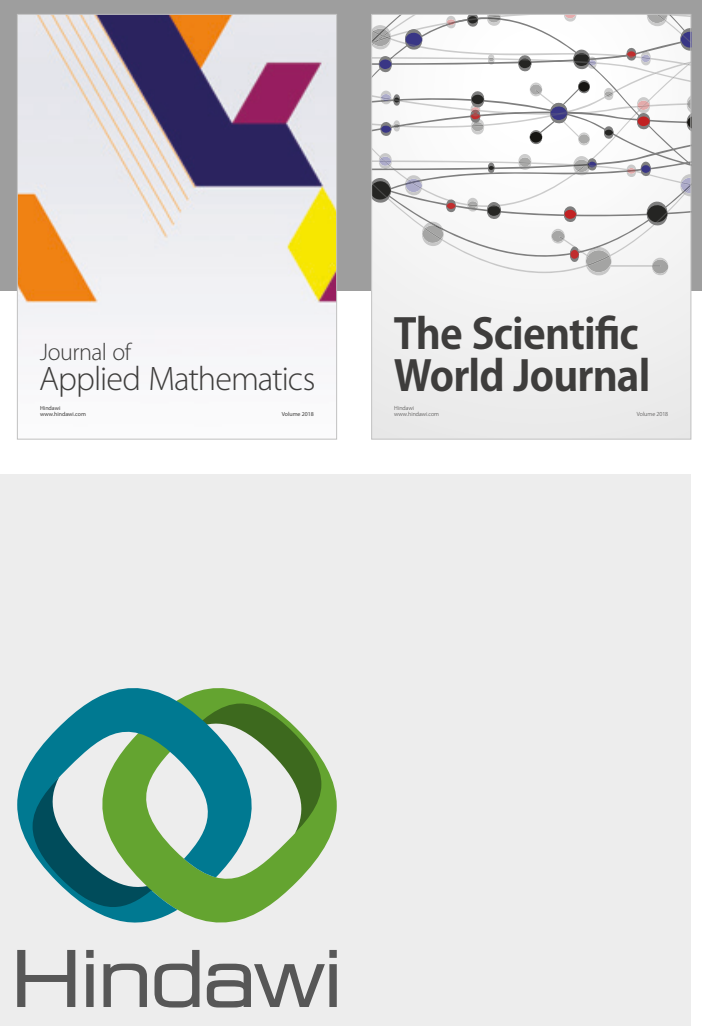

Submit your manuscripts at

www.hindawi.com

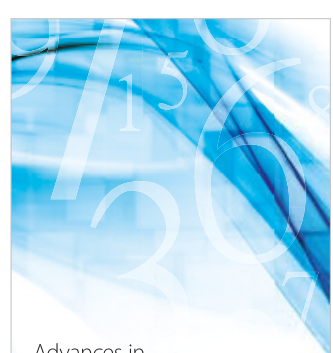

Advances in
Numerical Analysis
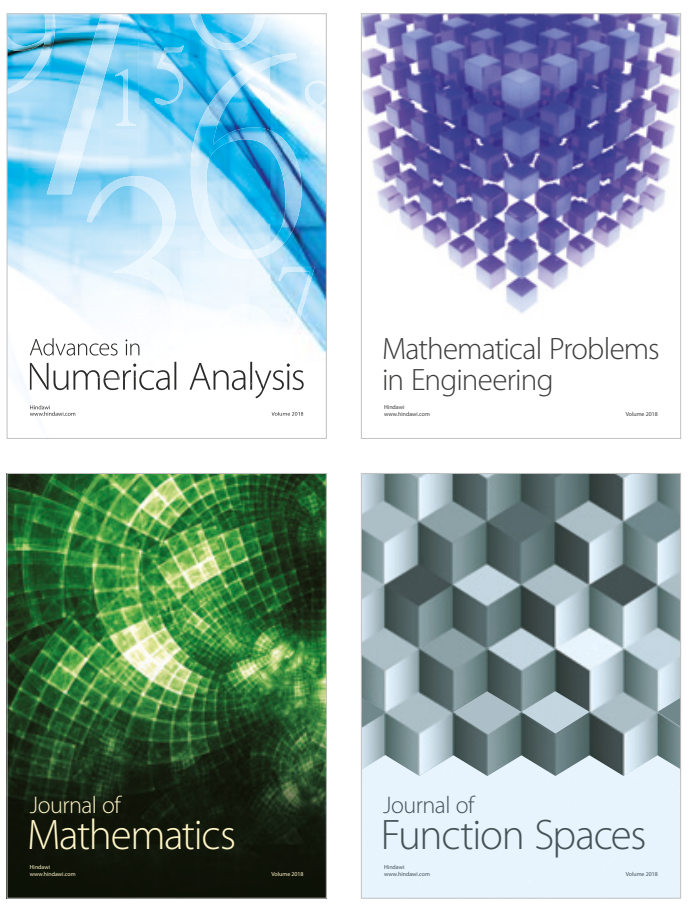

Mathematical Problems in Engineering

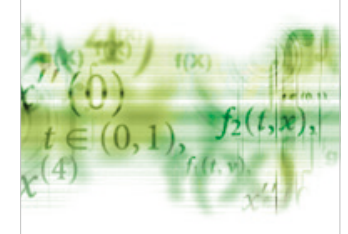

International Journal of

Differential Equations

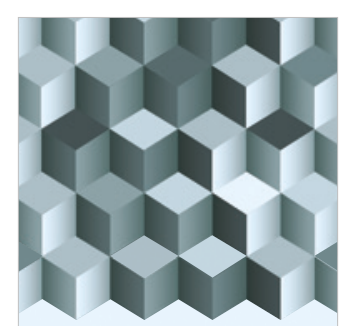

Journal of

Function Spaces

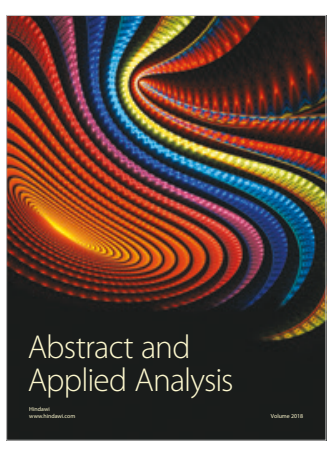

The Scientific

World Journal

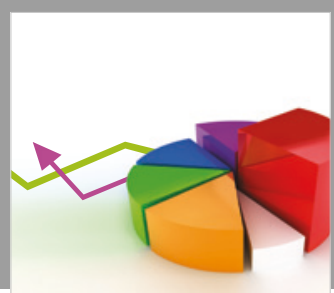

Journal of

Probability and Statistics
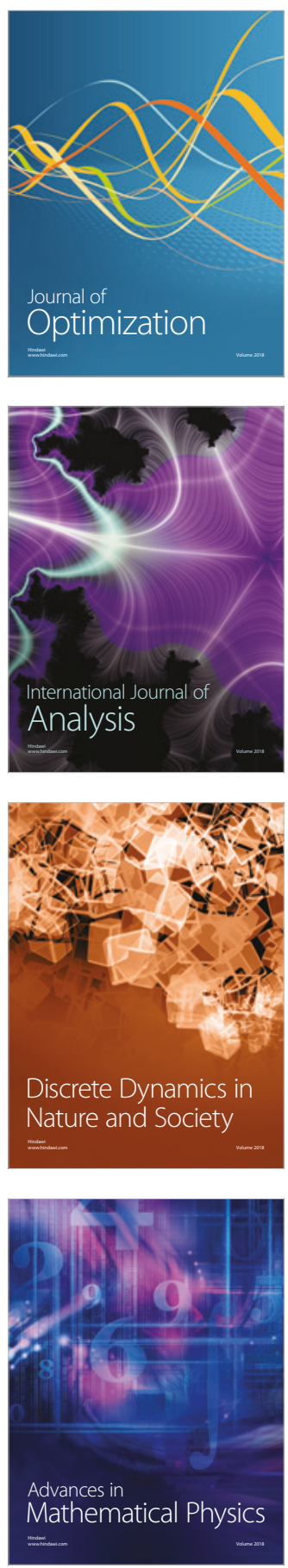